Natural Hazards and Earth System Sciences (2001) 1: 107-112

(C) European Geophysical Society 2001

\title{
Investigation of over-horizon VHF radio signals associated with earthquakes
}

\author{
Y. Fukumoto ${ }^{1}$, M. Hayakawa ${ }^{1}$, and H. Yasuda ${ }^{2}$ \\ ${ }^{1}$ Dept. of Electronic Engineering, University of Electro-Communications, 1-5-1 Chofugaoka, Chofu Tokyo 182-8585, Japan \\ ${ }^{2}$ Taiyo Musen Co., Ltd., Ebisu-nishi 2-20-7, Shibuya, Tokyo, Japan
}

Received: 7 May 2001 - Revised: 1 November 2001 - Accepted: 3 December 2001

\begin{abstract}
This paper presents the preliminary results on the possible reception of over-horizon VHF radio signals from a FM (frequency modulation) transmitter during abnormal situations (probably in close association with earthquakes). The transmitter is FM Sendai (frequency $=77.1 \mathrm{MHz}$ ) and the receiving station is our university (UEC) at Chofu, Tokyo, with the transmitter-receiver distance being about $310 \mathrm{~km}$. We first show a typical example of the reception of over-horizon VHF signals in which we present the amplitude behavior, azimuthal and incident angle estimation, etc. Then, we study the correlation between a signal anomaly and an earthquake and it seems that the over-horizon VHF signals observed are probably associated with earthquakes, observed about 7 to 0 days before an earthquake. The direction finding measurements have indicated that such signals are due to favorable tropospheric (but not ionospheric) conditions attributed to the effects of earthquakes.
\end{abstract}

\section{Introduction}

Electromagnetic phenomena have been recently considered to be very promising for short-term earthquake prediction (Hayakawa and Fujinawa, 1994; Hayakawa, 1999). One of the electromagnetic soundings is the use of pre-existing transmitter signals in different frequency ranges to investigate the seismo-atmospheric and seismo-ionospheric phenomena. Very convincing results relating to the seismoionospheric perturbations in the VLF and LF bands have already been reported (Gokhberg et al., 1989; Hayakawa et al., 1996; Molchanov and Hayakawa, 1998). This has led to an important scientific study of the lithosphere-atmosphereionosphere coupling. Biagi (1999) has also investigated the seismo-atmospheric effect in the sub-ionospheric LF (150$300 \mathrm{kHz}$ ) wave propagation.

Correspondence to: M. Hayakawa

(hayakawa@ aurora.ee.uec.ac.jp)
In the VHF frequency range, Kushida and Kushida (1998) have been working on the reception of over-horizon VHF transmitter signals (FM (frequency modulation) radio signals). They consider these signals to be associated with earthquakes and such receptions of the over-horizon VHF signals are due to the scattering by ionospheric perturbations caused by an earthquake. However, the mechanism is poorly understood. Due to this, we present our recent preliminary results in order to answer the fundamental questions regarding VHF radio sounding; (1) Can we actually receive an over-horizon VHF transmitter signal before an earthquake? and (2) What is the mechanism of reception for over-horizon VHF radio signals associated with earthquakes?

\section{Observation, receiving and antenna systems}

Figure 1 illustrates the relative location of the FM transmitter in Sendai (frequency $=77.1 \mathrm{MHz}$, power $=5 \mathrm{~kW}$, height $=193 \mathrm{~m}$, and horizontal polarization) (named FM Sendai) and the receiver at our university (UEC) (antenna height $=$ $33.8 \mathrm{~m}$ ). The distance between the transmitter and receiver is $312 \mathrm{~km}$ and the line of sight is $80 \mathrm{~km}$. Crosses in the figure indicate the epicenters of earthquakes with magnitudes greater than 3.5 during the period of our observation, which was five months, from 1 February to 30 June 2000. During this period midnight (00:00-05:30 LT), data could be used because during other time periods, the radio broadcasting (University of the Air) was transmitting on this same frequency in the Tokyo area.

The FM signal for one antenna system was received by a corresponding observing system, and was fed to a tuned HF amplifier. Then, the signal was frequency-translated to IF (intermediate frequency, $10.7 \mathrm{MHz}$ ) by means of a local oscillator. Afterwards, a part of the signal was rectified by a DAQ (data acquisition) board with a sampling rate of $10 \mathrm{~s}$ and analyzed by a PC (Fig. 2 is an example). Another part of the signal was fed to an IF amplifier and a detector, and the lower frequency component (demodulated signal) was 


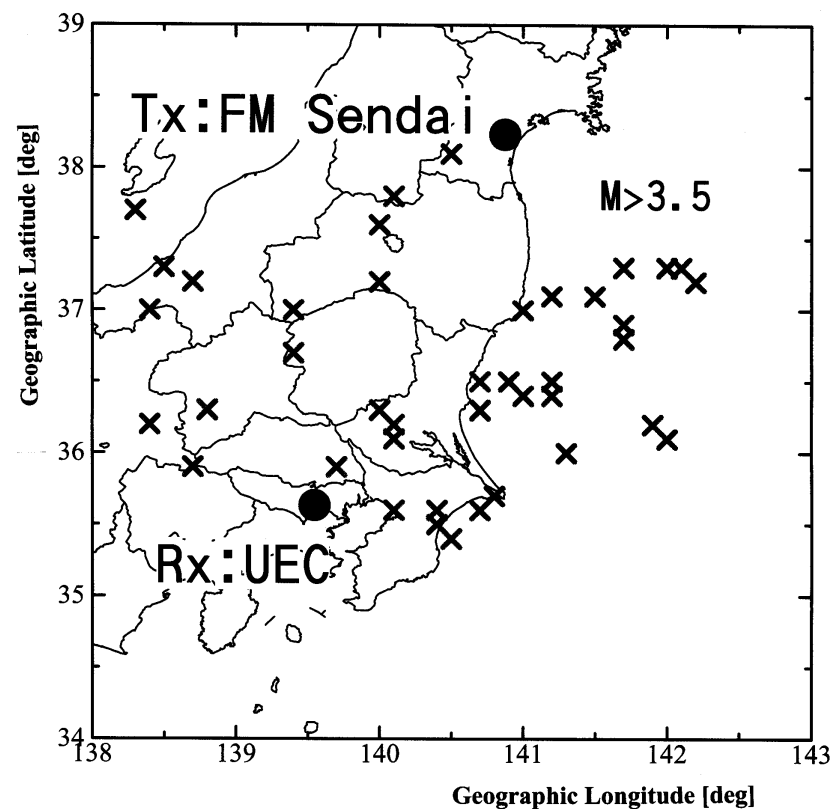

Fig. 1. Relative location of the FM transmitter in Sendai (FM Sendai) and the receiving station (our university), together with the locations of earthquake epicenters with magnitudes greater than 3.5 which occurred during the period of February through June 2000.

recorded on a video tape recorder to verify that the received signal was actually due to FM Sendai.

Next, we describe the antenna systems. Six Yagi antenna systems are used (each Yagi has 5 elements) and the receiving system for one antenna system is described above. As for the reception of a horizontally polarized signal, we have three different antenna systems with different bearing (azimuthal) angles of $-30^{\circ}, 0^{\circ},+30^{\circ}\left(0^{\circ}\right.$ means the direction between the receiver and transmitter; - (minus sign) indicates the west of the path and + (plus sign) the east of the path) for the fixed elevation angle of $0^{\circ}$ (horizontal). Additionally, we have three other antenna systems with three different elevation angles $\left(0^{\circ}, 45^{\circ}\right.$, and $90^{\circ}$ (vertical upward)) for the fixed azimuth of $0^{\circ}$ for the same horizontal polarization. We have one final antenna system to detect the signal with vertical polarization (bearing $=0^{\circ}$, elevation $=0^{\circ}$ ). By using the outputs from the two antennas directed in two azimuths $(\mathrm{S}(+)$ and $\mathrm{S}(-))$ (bearing $+30^{\circ}$ and $-30^{\circ}$, respectively) for the fixed elevation angle of $0^{\circ}$, we could estimate the azimuth of the observed signal by using the relationship of $\Theta(\operatorname{deg})=\left(180^{\circ} / \pi\right) \tan ^{-1}(\mathrm{~S}(+) / \mathrm{S}(-))-45^{\circ}$ and also the absolute amplitude (because the system is well calibrated).

\section{Observed results and correlation analysis}

On the normal days without any earthquake effects, we observe the background levels for all six antenna channels. However, when a certain effect (probably the effect of an earthquake) happens, we have various records based on previous observations, such as the one on 27 May 2000 as shown in Fig. 2. This was a typical example of the rectified IF output. Looking at the three panels (the two at the top right and one at the bottom left) directed at three different bearings (but with the same horizontal polarization and the same elevation angle of $0^{\circ}$ ), the most significant output was seen for the azimuth $+30^{\circ}$ (east of the path). By using the outputs from the two antennas $(\mathrm{S}(+)$ and $\mathrm{S}(-))$, we can locate the bearing direction, which has indicated that the over-horizon signals were found to have the bearing of about $+20^{\circ}$ (east of the path). Next, we discuss the incident angle of the signal by looking at the bottom three panels. As was easily seen from the results of the panels, the output for the elevation of $0^{\circ}$ is strongest, next for the elevation of $45^{\circ}$, and consequently, a negligible output was seen for the elevation of $90^{\circ}$ (vertically upward). By taking into account the radiation pattern in the elevation direction (as in the case of bearing estimation), the incident angle of the observed signal was estimated to be $\sim 10^{\circ}$. It was furthermore confirmed by listening to the demodulated low frequency signal of the observed signal that the sound signal of the observed VHF wave was precisely the one from FM Sendai, thus verifying reception of the overhorizon signal from FM Sendai. It seems that the reception of the abnormal over-horizon signal may have been a precursor to a big earthquake that occurred seven days later (on 3 June).

After having shown a typical example in Fig. 2, we want to know the possible correlation between such an anomalous signal (as in Fig. 2) and an earthquake. In order to study such a correlation, we take, as a reasonable quantity, an average of the signal amplitude (as in Fig. 2) during one particular day (i.e. about six hours during midnight hours as in Fig. 2) as $x(t)(t=$ day). On the other hand, another physical quantity was related to the earthquake. First, we deal with the earthquakes occurring east of the transmitter-receiver path (occurring within a radius of $300 \mathrm{~km}$ from the receiver with azimuths less than $120^{\circ}$ ) with magnitudes greater than 3.5. How to express another variable as $y(t)$ for any day providing us with earthquakes seems to depend on the mechanism of having such an anomalous VHF propagation. We assume that the magnitude of an anomalous VHF signal was proportional to the released energy of an earthquake $(E)(E$ $($ in erg $)=\exp (11.8+1.5 M))(M=$ magnitude $)($ GutenbergRichter law) (Kanamori, 1977), which seems reasonable and acceptable. The atmospheric perturbation effect in terms of an anomalous VHF signal amplitude was assumed to be related to the depth of an earthquake by the expression of $d^{-1}$ $(d=$ depth in $\mathrm{km})$, and finally, the signal intensity was reasonably related to the epicentral distance $(r$ in $\mathrm{km})$ as $r^{-1}$ which is accepted from the wave propagation theory, thus, $y=\exp (11.8+1.5 M) / d / r$. On some occasions when we have a few earthquakes on one particular day, we adopt the largest value $y$ for any earthquake as the daily value for $y(t)$. An alternative method was to take the sum of the $y$ 's corresponding to all earthquakes, but we have found no significant differences from the results. So, as an example, we indicate in Fig. 3 the daily plots of $x(t)$ and $y(t)$ for the earthquakes in the east of the path and for the signal intensity with a bearing 

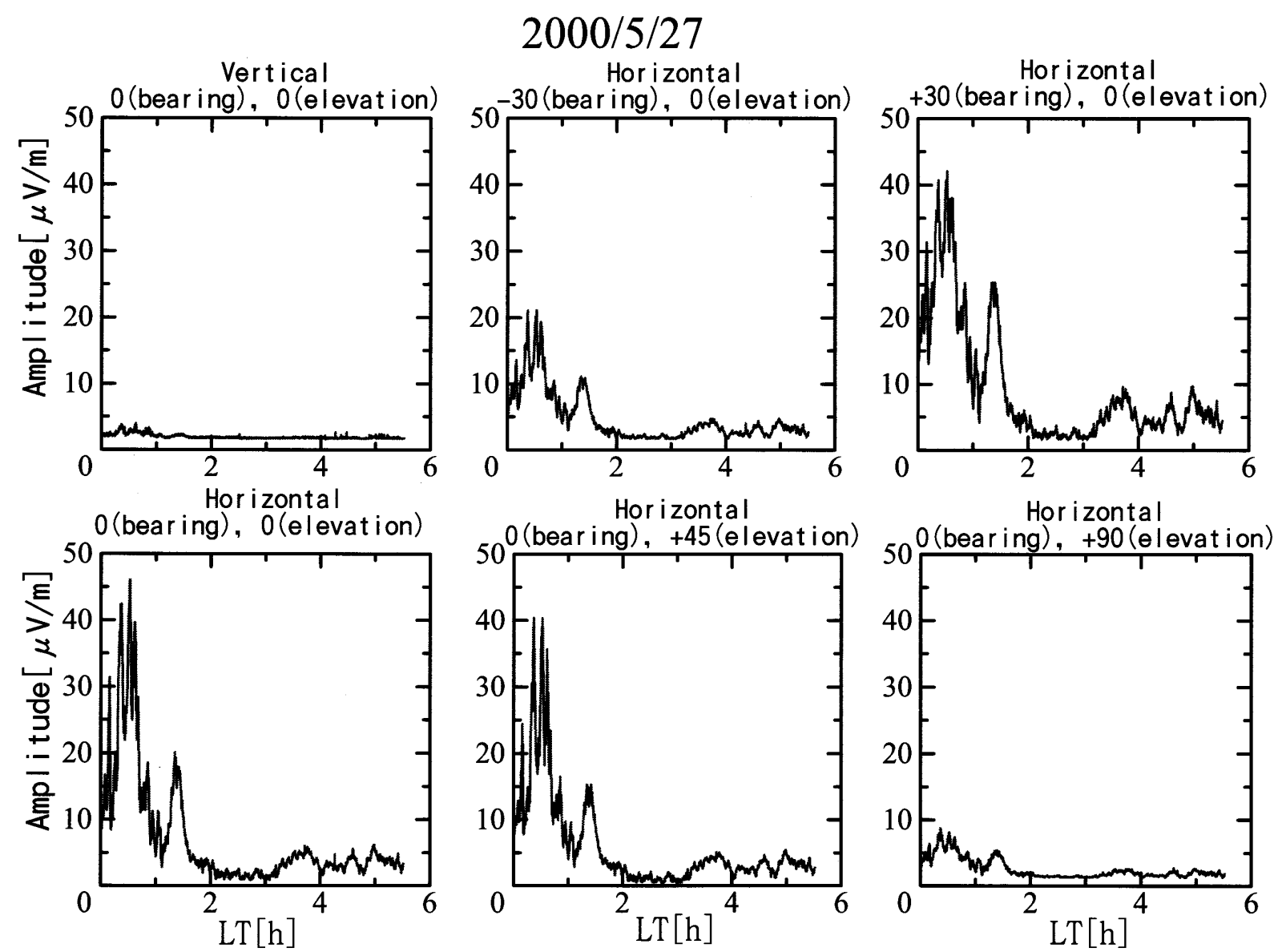

Fig. 2. An example of abnormal receptions of over-horizon FM signals on 27 May 2000. The outputs from six different antenna systems are illustrated; from left to right on the upper panel; (1) vertical polarization (bearing $=0^{\circ}$, elevation $=0^{\circ}$ ), (2) horizontal polarization $($ bearing $=-$ $30^{\circ}$, elevation $=0^{\circ}$ ), (3) same polarization (bearing $+30^{\circ}$, elevation $=0^{\circ}$ ), then on the lower panel, from right to left; (4) horizontal polarization (bearing $=0^{\circ}$, elevation $\left.=0^{\circ}\right)(5)$ same polarization (bearing $=0^{\circ}$, elevation $\left.=45^{\circ}\right)$ and $(6)$ same polarization $\left(\right.$ bearing $=0^{\circ}$, elevation $=90^{\circ}$ (upward)).

of $+30^{\circ}$ and elevation of $0^{\circ}$ (horizontal polarization), corresponding to the top third panel in Fig. 2. When we look at Fig. 3 and compare these two plots, we can infer the correlation between the two. We look at the periods when the signal intensity exceeds a threshold (say $4 \mu \mathrm{V} / \mathrm{m}$ ), and we try to make group-to-group correlations between the two. At the beginning of February we had several days with enhanced signal intensity (reception of over-horizon signal), and during that period we had earthquakes correspondingly. A series of earthquakes in the early half of May were likely to be preceded by enhanced VHF signals in the beginning of May. Similarly, significant enhancement at the end of May (as in Fig. 2) was likely to be a precursor to the big earthquake at the beginning of June. A significant peak around 20 April seems to be closely associated with small earthquakes at the end of April. Earthquakes at the beginning of March and at the beginning of April were not clearly preceded by any VHF signal enhancements.

Apart from this kind of group-to-group correlation, we feel that there seems to be some kind of one-to-one correla- tion existing between the propagation anomaly and an earthquake when we look at the observational data daily, i.e. the correlations between a local maximum (as in Fig. 3) and an earthquake. Although the application of this method for such data was not verified, we performed a cross-correlation analysis. Figure 4 is the corresponding result (lag-correlogram, though not normalized), for our six antenna systems. Let us look at the results for the antenna system (horizontal polarization, bearing of $0^{\circ}$ and elevation of $0^{\circ}$ ) (down left in Fig. 4). A minus sign in the figure means that the signal reception was taking place before an earthquake. The figure indicates that there is a broad maximum from -7 days to 0 days, which may suggest that such receptions of over-horizon signals tends to occur before an earthquake.

The abnormal signal on 27 May in Fig. 2 was likely to be a precursor to a big earthquake $(M=5.8)$ which occurred on 3 June, whose epicentral azimuth was $+94^{\circ}$. However, the direction finding gave us the result of $+20^{\circ}$ for the event in Fig. 2, and there seems to be a significant difference between the observed signal bearing and epicentral direction. Based 


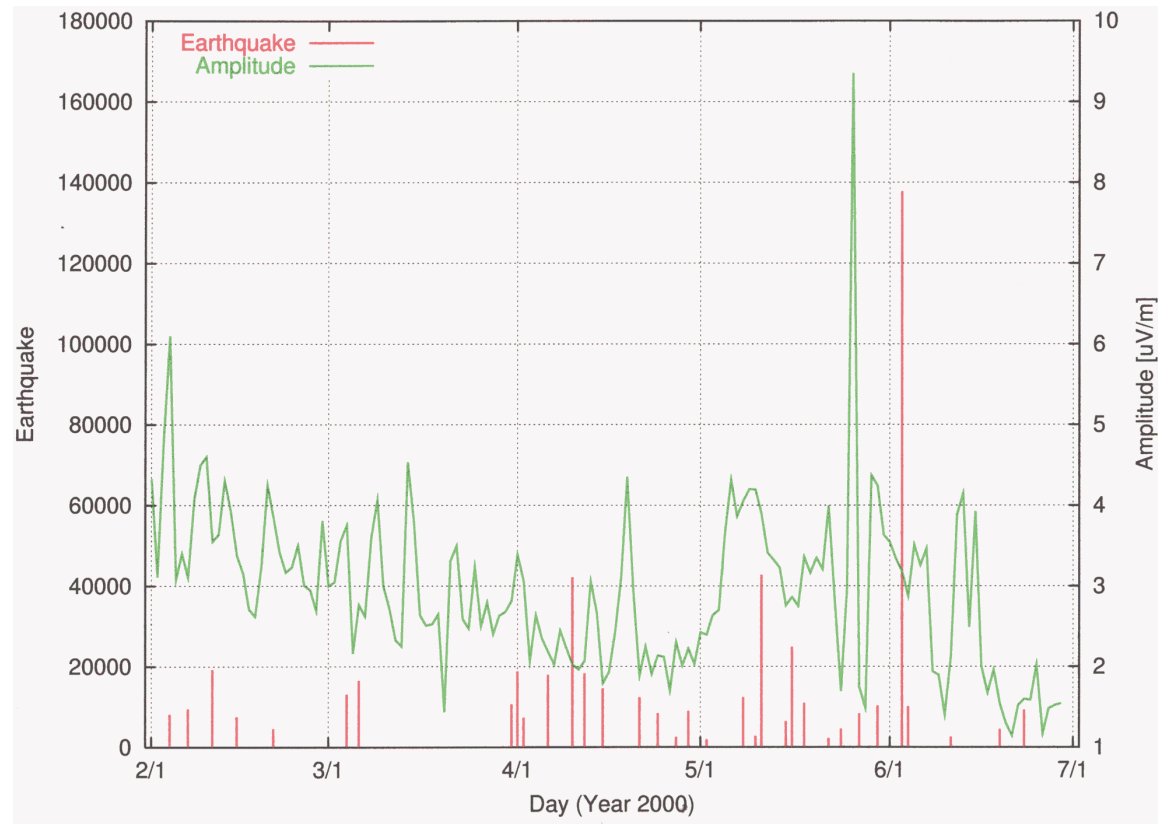

Vertical
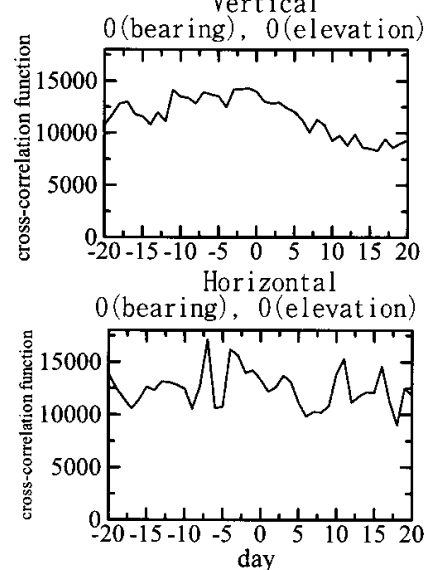

Horizontal
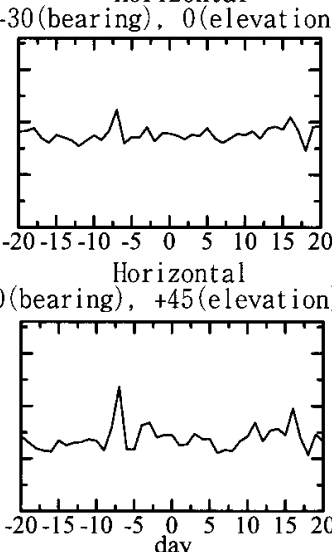

Hor izontal

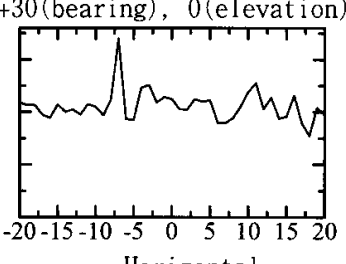

Horizontal

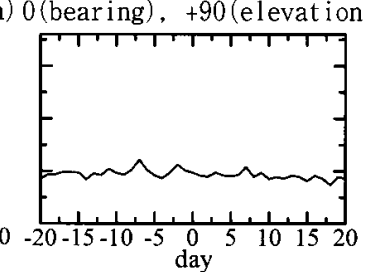

Fig. 3. Temporal plots of the VHF signal amplitude and the corresponding information on earthquakes during the entire observation period of five months.

Fig. 4. Cross-correlation between the earthquakes taking place in the east of the path and abnormal signal reception (for the same antenna systems as in Fig. 2). Minus sign on the abscissa indicates that the signal reception was occurring before an earthquake.

on the correlation in Fig. 4, we have identified the earthquake corresponding to each propagation anomaly during the period of -7 days to 0 days. The statistical analysis on such a difference between the signal bearing and the corresponding epicentral direction is summarized in Fig. 5, indicating that the signal bearing is always more westward than the epicentral direction (the maximum difference is peaked at $\sim 30^{\circ}$ ). Figure 5 suggests that the signal was coming very close to the coast of the Japan Islands, which may indicate the presence of a large perturbed region associated with the earthquake. We can comment on the statistical result of the signal elevation, which indicates that the signal elevation was less than $20^{\circ}$, while peaking at about $10^{\circ}$.

Figure 6 illustrates the intensity of the observed overhorizon VHF signals, compared with the theoretical expectation (Hall et al., 1996). The full line is the theoretical curve for the expected signal amplitude based on the information according to the transmitter characteristics (antenna power, etc.) and receiver height. Up to the distance of the line of sight ( $~ 50-80 \mathrm{~km})$, the wave is considered a space wave and beyond this critical distance we have only a diffracted wave (indicated by a full line in the lower part of the figure), while the upper full line indicates the free space wave intensity. The observed signal intensity was mainly below the broken line (which is the extrapolaration of the space wave propagation), but the strongest intensity exceeded this level.

\section{Conclusion and discussion}

The following observational facts have emerged from our preliminary analysis over five months.

1. Over-horizon VHF transmitter signals (though they are not received during normal conditions) were sometimes 

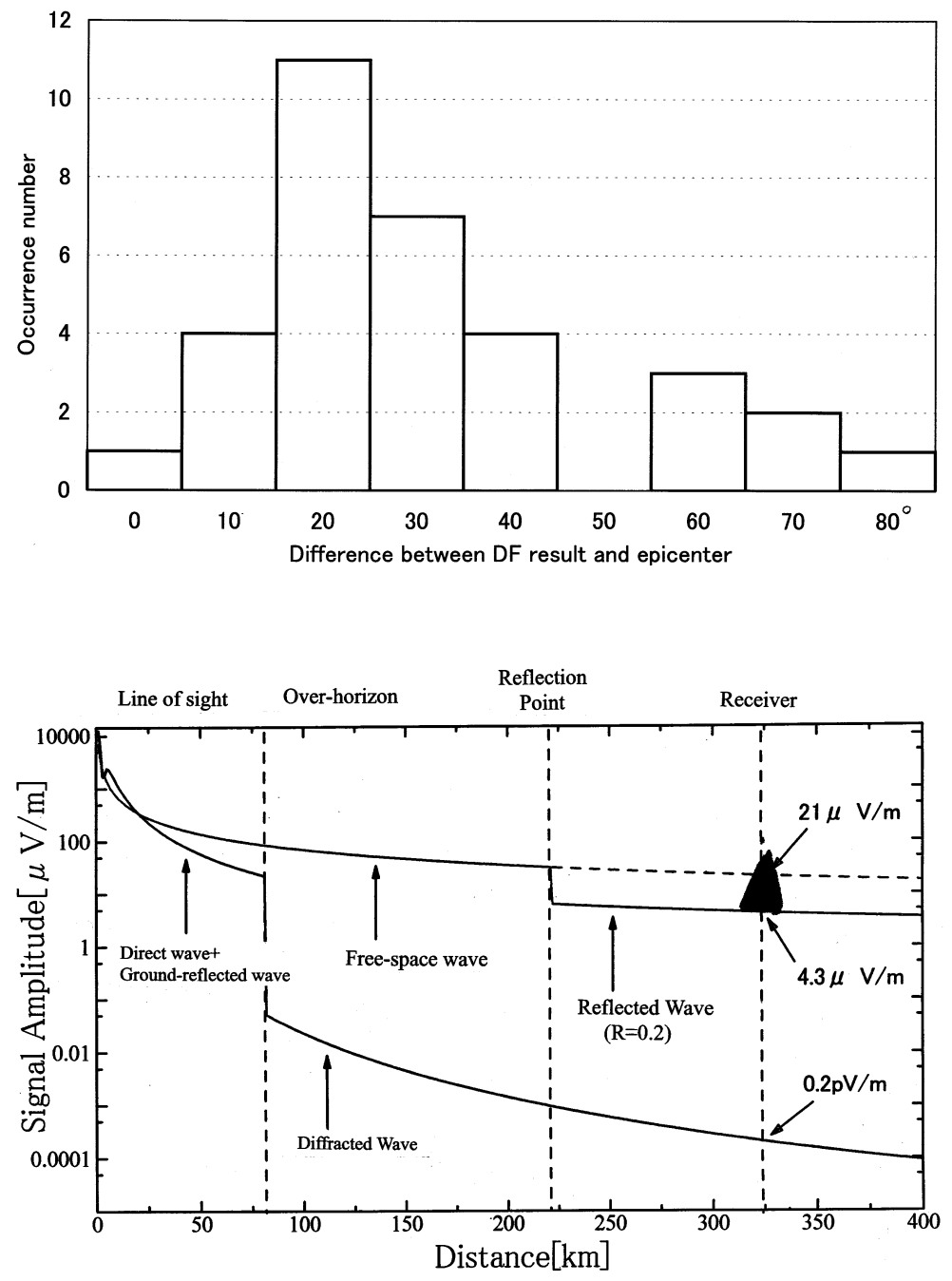

Fig. 5. Statistical plot of the differences between the signal bearings and epicentral directions in the case of earthquakes occurring only in the east of the greatcircle path. Signal was always coming more westward than the epicentral direction.
Fig. 6. Theoretical estimation of signal intensity as a function of distance, together with the observed signal intensity. observed during abnormal conditions (probably associated with earthquakes).

2. Such abnormal over-horizon VHF signals were found to be received at our observing station with a small incident angle (incident angle $<20^{\circ}$ ).

3. The direction finding of the bearing of observed overhorizon signals showed that there was sometimes substantial differences with regards to the bearing of a future epicenter.

4. The correlation between the abnormal over-horizon VHF signals and earthquakes indicated that there was a broad maximum about 7 to 0 days before an earthquake, suggesting a precursory effect in VHF signal reception.

Based on these preliminary observational facts, we tried to answer the fundamental questions raised in the Introduction. First of all, the signal from FM Sendai was actually observed on some occasions at a receiving station beyond the horizon. A comparison of the daily variations concerning the
VHF signal amplitude with that of earthquakes and the crosscorrelation between the two, may indicate that such a reception of over-horizon VHF signals might be due to the effect of earthquakes. The various over-horizon signals were observed with a small elevation angle (around $10^{\circ}$, and less than $20^{\circ}$ ), which may imply that the signal reception was due to tropospheric effects (definitely not due to ionospheric scattering). Also, the bearing of the observed signal was significantly different from the future epicentral direction, and we may infer that the perturbed region was considerably large (with radius of $100 \mathrm{~km}$ based on considerations of the signal bearing and the epicentral position) and the signal was likely to be coming from the coastal region. The important point was that the signal intensity was much higher than the diffracted level and was significantly large as shown in Fig. 6. Consequently, this means that the signal reception was unlikely caused by ionospheric scattering, but due to very favorable tropospheric propagation conditions associated with an earthquake.

With future work, it is of essential importance to show convincing evidence and also to perform appropriate correlation studies between abnormal signal receptions and earth- 
quakes to demonstrate the correlation between the two. Also, we need to investigate the generation mechanism of atmospheric perturbation associated with earthquakes. Finally, we have to investigate the natural noise (for vertical polarization in Figs. 2 and 4) associated with earthquakes.

Acknowledgements. The authors would like to express their sincere thanks to T. Yoshida of Hiroshima City University for his assistance in the system design.

\section{References}

Biagi, P.: Seismic effect on LF radio waves, in: Atmospheric and Ionospheric Electromagntic Phenomena Associated with Earthquakes, (Ed) Hayakawa, M., Terra Sci. Pub. Co., 535-542, 1999.

Gokhberg, M. B., Gufeld, I. L., Rozhnoy, A. A., Marenko, V. F., Yampolsky, V. S., and Ponomaren, E. A.: Study of seismic influence on the ionosphere by super long-wave probing of the Earthionosphere waveguide, Phys. Earth Planet. Inter., 57, 64, 1989.
Hall, M. P., Barclay, L. W., and Hewitt, M. T.: Propagation of Radiowaves, Inst. Electr. Engrs., London, UK, 1996.

Hayakawa, M., (Ed): Atmospheric and Ionospheric Electromagnetic Phenomena Associated with Earthquakes, Terra Sci. Pub. Co., Tokyo, 997, 1999.

Hayakawa, M. and Fujinawa, Y. (Eds): Electromagnetic Phenomena Related with Earthquakes, Terra Sci. Pub. Co., Tokyo, 667, 1994.

Hayakawa, M., Molchanov, O. A., Ondoh, T., and Kawai, E.: The precursory signature of Kobe earthquake on VLF subionspheric signal, J. Comm. Res. Lab., 43, 169-180, 1996.

Kanamori, H.: The energy release in great earthquakes, J. Geophys. Res., 82, 2981-2987, 1977.

Kushida, Y. and Kushida, R.: On the possibility of earthquake forecast by radio observation in the VHF band, RIKEN Review, 19, 152-160, 1998.

Molchanov, O. A., and Hayakawa, M.: Subionospheric VLF signal perturbations possibly related with earthquakes, J. Geophys. Res., 103, 17 489-17 504, 1998. 